\title{
Nanomaterials: Electrical, Magnetic, and Photonic Applications
}

\author{
TERRY T. XU ${ }^{1,3}$ and JUNG-KUN LEE ${ }^{2}$ \\ 1.-Department of Mechanical Engineering and Engineering Science, The University of North \\ Carolina at Charlotte, Charlotte, NC 28223, USA. 2.-Department of Mechanical Engineering and \\ Materials Science, University of Pittsburgh, Pittsburgh, PA 15261, USA. 3.-e-mail: ttxu@uncc. \\ edu
}

The nanomaterials section of the April issue of $J O M$ has a unique combination of short review papers and research papers. These papers present the recent progress on controlled synthesis of magnetic nanoparticles (MNPs), the discussion of a fundamental physics concept of quantum capacitance and its applications in nanoelectronics, and the exploration of novel Ti: $\mathrm{Fe}_{2} \mathrm{O}_{3}-\mathrm{SnO}_{2}$ nanostructured photoanodes for efficient photoelectrochemical (PEC) water splitting.

MNPs have been extensively studied in the last few decades due to their potential applications in data storage, biomedical uses, and other possibilities. It is well known that the physicochemical properties of MNPs are size, shape, and crystallinity dependent. Therefore, for future mass applications, one key challenge is to explore reliable, scalable, and economical synthesis methods to produce MNPs with well-defined microstructures that allow for high permittivity and/or ferromagnetism at room temperature. The article "Unusual Function of Modified Polyolefins for Manipulating Magnetic Nanostructures" contributed by Qingliang He, Tingting Yuan, Suying Wei, and Zhanhu Guo reviews the usage of polypropylene-graftmaleic anhydride (PP-g-MA) as a polymeric surfactant for controlled synthesis of MNPs. PP-g-MA is widely used in industry as a well-known plastic additive to stabilize the inorganic materials in a polymer matrix because the PP-group and MA-group play complementary roles. Compared with other commonly used small-molecular-weight surfactants, PP-g-MA has certain advantages such as its unexpected controlling ability of the crystallinity of as-synthesized MNPs, and its compatibility with many polymers for future in situ preparation of magnetic polymer nanocomposites with desirable physicochemical properties.

In nanoelectronics, capacitors play a critical role. Nanocapacitors have demonstrated strong size

Terry T. Xu and Jung-Kun Lee are the guest editors for the Nanomaterials Committee of the TMS Functional Materials Division (FMD) [formerly the Electronic, Magnetic \& Photonic Materials Division (EMPMD)], and coordinators of the topic Nanomaterials: Electrical, Magnetic and Photonic Applications in this issue. effects, and the observed phenomena sometimes do not obey classic electrostatic theory. The article "Quantum Capacitance: A Perspective from Physics to Nanoelectronics" contributed by Yuranan Hanlumyuang and Pradeep Sharma reviews the concept of quantum capacitance: its physical origins and its future applications in tailoring the performance of nanoelectronics. By applying the idea of negative quantum capacitance, novel heteronanostructures with anomalously large capacitance have been recently demonstrated by both experiments and numerical simulations. Furthermore, it has been predicted that both the sign and the value of quantum capacitance can be tuned by small external strains. This prediction could open new ways for future fabrication of nanoelectronics with desired functions.

PEC water splitting aims at the effective capture of sunlight and subsequent conversion into fuels such as hydrogen. This energy conversion technique, if made cheaply and with a high enough conversion efficiency, could have a strong impact on future energy conversion and storage industries. One very promising material for cheap PEC cells is $\mathrm{Fe}_{2} \mathrm{O}_{3}$, which has superior optical properties for solar energy harvesting. However, its low carrier mobility is a critical weakness. To address this problem, one solution is to use nanostructured photoelectrodes to increase the hole collection distance and shorten the light penetration path. The article "Heterojunction $\mathrm{Fe}_{2} \mathrm{O}_{3}-\mathrm{SnO}_{2}$ Nanostructured Photoanode for Efficient Photoelectrochemical Water Splitting" contributed by Hyun Soo Han, Sun Shin, Jun Hong Noh, In Sun Cho, and Kug Sun Hong reports their recent efforts to use heterojunction $\mathrm{Fe}_{2} \mathrm{O}_{3}-\mathrm{SnO}_{2}$ nanostructured photoanodes for efficient PEC water splitting. The photoanode architecture prepared by drop casting and laser ablation methods consists of light-absorbing Ti-doped $\mathrm{Fe}_{2} \mathrm{O}_{3}$ nanoparticles and carrier-delivering $\mathrm{SnO}_{2}$ nanostructured film with a large surface area and open channels. The performance of this novel photoanode system shows a $34 \%$ increase in photocurrent density compared with a Ti: $\mathrm{Fe}_{2} \mathrm{O}_{3}$ flat photoanode. 